\section{Retinal angiomatous proliferation or retinal anastomosis to the lesion}

AW Scott and SB Bressler

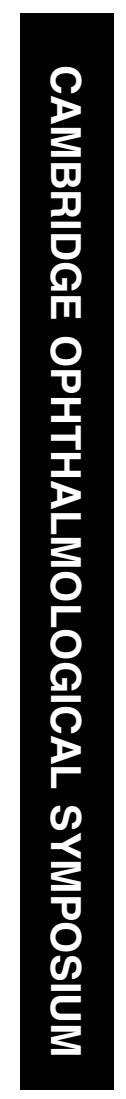

physical relationship to the retinal pigment epithelium (RPE). ${ }^{4}$ Type 1 neovascularization describes fibrovascular tissue that is posterior to the RPE, whereas Type 2 neovascularization refers to a fibrovascular complex that lies anterior to the RPE. Freund has proposed modifying the original Gass classification to add Type 3 neovascularization to refer to intraretinal neovascularization, as is suspected to occur in these RAP lesions. ${ }^{5,6}$

Yannuzzi et al. introduced the term 'retinal angiomatous proliferation' to describe a vascular process that these authors believe originates within the neurosensory retina, beginning with capillary proliferation, formation of intraretinal neovascularization, and retinal-retinal anastamoses. This retinal neovascularization then extends beneath the neurosensory retina to become subretinal neovascularization (SRN). With time, the SRN may merge with the choroidal circulation beneath the RPE to form retinal-choroidal anastomosis. ${ }^{7}$ However, the vasogenic sequence of RAP lesions remains controversial. In contrast to the hypothesis offered by the Yannuzzi et al. we believe, as did Gass, ${ }^{8}$ that choroidal neovascularization (CNV) is the initiating event of a RAP lesion rather than retinal neovascularization. Typically, this lesion begins as an occult CNV lesion without a classic component that is then followed by a retinal vessel diving posteriorly into the lesion and communicating with the $\mathrm{CNV}$ lesion within the subretinal space. For this reason, we prefer to refer to this entity as 'retinal anastomosis to the lesion' or RAL. For simplicity and consistency, however, we will refer to this condition as 'RAP' throughout this manuscript.

Just as the origin of RAP lesions remains controversial, so does its natural history. RAP lesions have been characterized in the literature as having a poor natural history, ${ }^{9}$ but it is unclear what the reference group is.
Retina Division, Department of Ophthalmology, Wilmer Eye Institute, Johns Hopkins University School of Medicine, Baltimore, MD, USA

Correspondence: SB Bressler, Retina Division, Department of Ophthalmology, Wilmer Eye Institute, Johns Hopkins University School of Medicine, 600 North Wolfe Street, Maumenee 706, Baltimore, MD 21287, USA

Tel: + 4109553648 .

Fax: +410 6143011 .

E-mail: sbressler@jhmi.edu

Received: 3 November 2009 Accepted: 17 November 2009

Published online:

18 December 2009

This article was presented at the 2009 Cambridge Ophthalmological

Symposium 
Is the natural history truly distinct from other neovascular AMD presentations? It is also unclear whether these statements refer to vision outcomes, anatomic outcomes, or both. Another recurring theme within the literature reporting on RAP lesions is that these lesions do not respond well to treatment, and that no definite therapy has been shown to be beneficial at reducing visual loss and controlling the lesion. This has led to the use of a variety of treatment modalities for these lesions, with the list of therapies resembling those that have been used for any AMD-related CNV lesion, including direct laser photocoagulation, transpupillary thermotherapy, surgical removal of the lesion, surgical excision of the retinal feeder vessels, photodynamic therapy (PDT) guided by fluorescein or indocyanine green (ICG) dye with and without intravitreal triamincolone, periocular anecortave acetate, antivascular endothelial growth factor (VEGF) regimens with pegaptanib sodium (EyeTech Pharmaceuticals, New York, USA), ranibizumab (Lucentis, Genentech Inc, South San Francisco, CA, USA), or bevacizumab (Avastin, Genentech Inc., South San Francisco, CA, USA), or various combinations of the above. ${ }^{10}$ Within this review, we will explore the data surrounding the natural history of RAP and what we have learned about how this condition responds to treatment.

\section{Natural history of RAP lesions}

The prospective data on the natural history of RAP lesions can be found within one of the phase III trials evaluating PDT, the Verteporfin in Photodynamic Therapy (VIP) trial (Visudyne, Novartis Pharma AG, Basel, Switzerland). ${ }^{11}$ This was a double-masked, placebo-controlled, randomized, multicenter clinical trial that enrolled adults with a minimum age of 50 years, who had subfoveal CNV from AMD. Of the 339 eyes studied in the VIP study, roughly 75\% (258/339) had subfoveal occult lesions without evidence of classic CNV. These individuals were required to have entry vision of at least 20/100, lesion size of up to 9 disc areas (DAs), and evidence of recent disease progression. Recent disease progression was considered to be present if any of the following was true: (1) blood associated with the $\mathrm{CNV}$ lesion was present, (2) vision loss of at least one line of acuity had occurred in the presence of known $\mathrm{CNV}$, or (3) at least a $10 \%$ growth in the greatest linear diameter of the lesion had occurred within 3 months of study entry. ${ }^{11}$ The presence of RAP was not an exclusion criterion for VIP study participation.

After reporting the VIP trial results, the baseline stereoscopic film-based colour fundus photographs and fluorescein angiograms were reviewed by the Wilmer Reading Center for the presence or absence of RAP. The

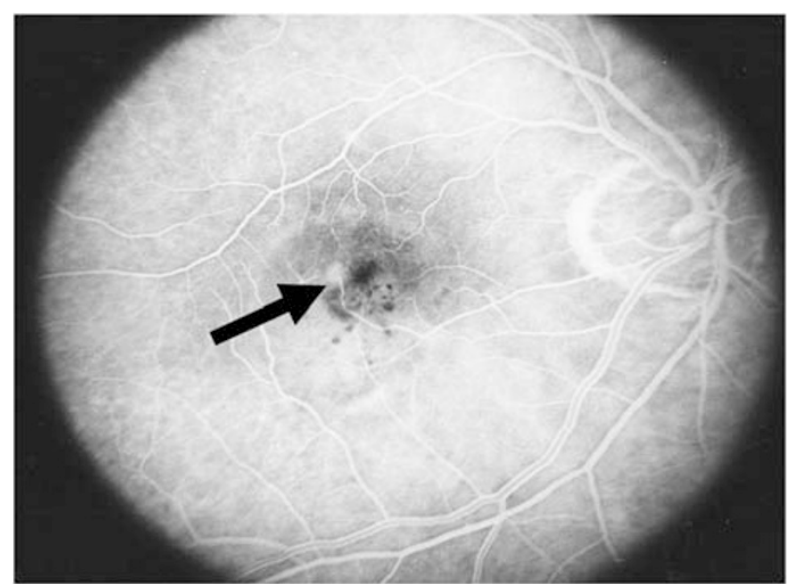

Figure 1 Mid-phase fluorescein angiogram shows a retinal vessel (black arrow) diving at a $90^{\circ}$ angle from the retina towards the RPE.

reading center graders remained masked to all vision and anatomic outcomes when making this determination. Eyes were considered to have a RAP lesion if either of the two following criteria was met: (1) a retinal vessel extended at a right angle from the inner retina towards the RPE anterior to a CNV lesion (Figure 1), or (2) a single intraretinal haemorrhage or a cluster of intraretinal haemorrhages were present overlying a retinal vessel, which itself was directly anterior to CNV (Figure 2). ICG angiography was not used to identify RAP lesions within this study.

To address the questions surrounding the natural history of RAP, we will examine vision outcomes in the subgroup of VIP participants who had RAP and were assigned to the placebo arm of the trial, and we will compare this group with the remaining natural history eyes that did not have RAP at study entry. Overall, 10\% (27/258) of the VIP study eyes with occult CNV lesions were categorized as RAP lesions, with approximately equal representation of RAP lesions in both the PDT and natural history arms of the trial $(10 \%(17 / 166)$ vs $11 \%$ (10/92), respectively). Although only 10 eyes in the natural history group had RAP lesions, the data collected on these individuals were prospective, followed standardized methods, and were complete through 24 months; hence, it provides meaningful information that cannot be found elsewhere, while also offering the concurrently enrolled comparison groups of natural history eyes without RAP and eyes with RAP treated with PDT. Table 1 summarizes select baseline features of RAP-containing lesions. Eyes with RAP tended to have a smaller CNV lesion size, as 21/27 (78\%) RAP eyes had lesions $\leqslant 4$ DAs compared with $98 / 228$ (43\%) of the lesions without RAP. Thus, smaller lesion size may be a characteristic of RAP lesions relative to other AMD 

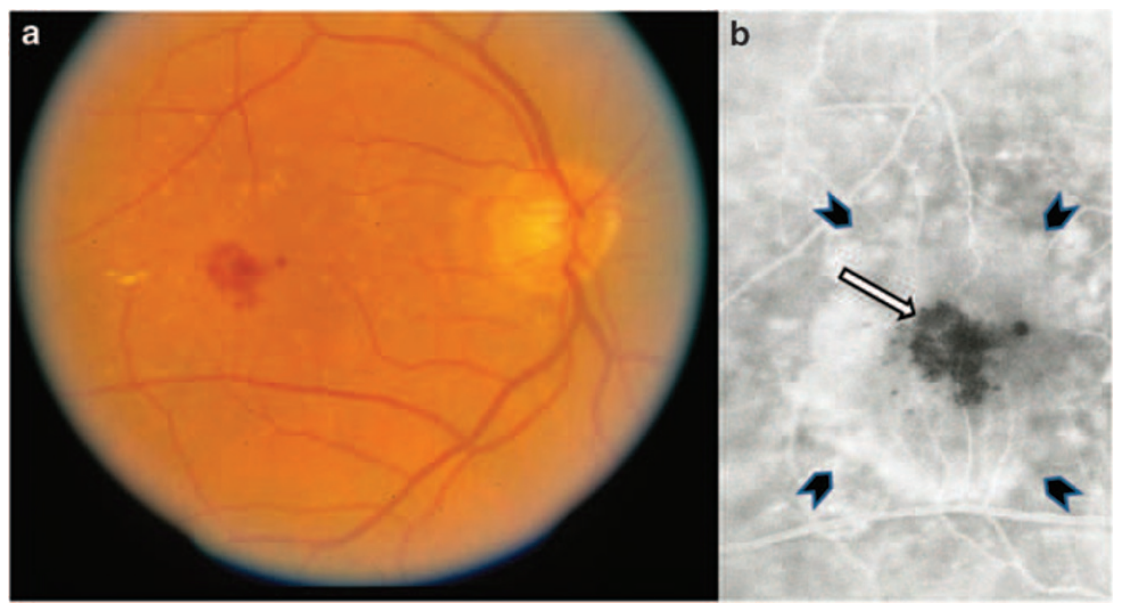

Figure 2 (a) Colour fundus photograph shows a cluster of intraretinal haemorrhages that overlie a retinal vessel. (b) Mid-phase fluorescein angiogram shows the cluster of intraretinal haemorrhages blocking fluorescence from the retinal artery that lies beneath the cluster (white arrow). The haemorrhage and the obscured retinal vessel lie anterior to an occult CNV lesion without a classic component (arrowheads).

Table 1 RAP by baseline variables-occult lesions with no classic components

\begin{tabular}{|c|c|c|}
\hline Variable & RAP present & RAP absent \\
\hline Verteporfin arm & 17 & 149 \\
\hline Placebo arm & 10 & 82 \\
\hline Lesion $\leqslant 4$ DAs & $21(78 \%)$ & $98(43 \%)$ \\
\hline Lesion $>4$ DAs & $6(22 \%)$ & $130(57 \%)$ \\
\hline Visual acuity $20 / 20-20 / 50^{+2 a}$ & $6(22 \%)$ & $121(52 \%)$ \\
\hline Visual acuity $20 / 50^{+1}-20 / 100^{\mathrm{b}}$ & $21(78 \%)$ & $110(48 \%)$ \\
\hline
\end{tabular}

lesions in general, which may imply that they have greater potential for further growth. In terms of presenting level of visual acuity, vision was categorized into two strata: the better level of vision included those with vision of $20 / 20$ through to $20 / 50$, whereas the lower vision group were those with 20/50 through to 20/100 visual acuity. The majority of the RAP eyes were in the lower vision strata $(78 \%)$ at presentation, relative to $50 \%$ of the eyes without RAP. This observation is consistent with the clinical impression shared by many authors that RAP lesions have a more ominous course. ${ }^{12}$

Table 2 summarizes several visual acuity outcomes at the 24-month visit in the VIP trial, such as, vision loss of three or more lines of acuity, vision loss of at least six lines of acuity, visual acuity of 20/200 or worse, and mean change in visual acuity from baseline. In three of these four analyses, the natural course for occult CNV lesions without a classic component with RAP appears very similar to similar lesions without RAP. Note that mean visual acuity loss was -5.3 lines in RAP eyes as compared
Table 2 Visual acuity outcomes with or without RAP (occult lesions with no classic components)

\begin{tabular}{lcc}
\hline Outcome & $\begin{array}{c}\text { RAP present } \\
\text { placebo }(\mathrm{n}=10)\end{array}$ & $\begin{array}{c}\text { RAP absent } \\
\text { placebo }(\mathrm{n}=82)\end{array}$ \\
\hline$\geqslant$ 3-line visual acuity loss & $90 \%$ & $66 \%$ \\
$\geqslant$ 6-line visual acuity loss & $40 \%$ & $48 \%$ \\
Visual acuity $\geqslant 20 / 200$ & $50 \%$ & $44 \%$ \\
Mean visual acuity change & -5.3 lines & -5.1 lines \\
\hline
\end{tabular}

with -5.1 lines in non-RAP CNV eyes at 24 months. Although there was one analysis in which eyes without RAP had a better natural history (vision loss of at least three lines of acuity), the totality of the evidence would suggest that there is no dramatic difference in the natural history of eyes with RAP compared with those without.

\section{Do eyes with RAP lesions respond differently to treatment for CNV?}

Another controversy surrounding RAP lesions is whether or not they favourably respond to treatment. As an example, Bottoni et al. reported that no information from the VIP trial is available that describes the use of PDT for the treatment of RAP lesions, and they referenced an abstract that reported that PDT is of little benefit in these lesions. ${ }^{13,14}$

Table 3 shows 2-year vision outcomes of the VIP trial participants with occult lesions without a classic component by treatment assignment (PDT vs sham), subgrouped by the presence or absence of RAP. For each visual acuity outcome, RAP lesions treated with PDT always fared better than eyes with RAP lesions assigned to the sham arm. The same is true for occult lesions without 
Table 3 Visual acuity outcomes with or without RAP (occult lesions with no classic components; baseline to month 24 examination)

\begin{tabular}{lcc}
\hline Outcome & $\begin{array}{c}\text { RAP present } \\
\text { vert }(\mathrm{n}=17)\end{array}$ & $\begin{array}{c}\text { RAP absent } \\
\text { vert }(\mathrm{n}=149)\end{array}$ \\
\hline 3-line visual acuity loss & $59 \%$ & $54 \%$ \\
$\geqslant$ 6-line visual acuity loss & $35 \%$ & $28 \%$ \\
Visual acuity $\geqslant 20 / 200$ & $47 \%$ & $26 \%$ \\
Mean visual acuity change & -4.4 lines & -3.7 lines \\
\hline
\end{tabular}

RAP treated with PDT relative to the sham group. The magnitude of the treatment effect seems to be greater for eyes without RAP compared with eyes with RAP; however, with only 27 total RAP eyes, the confidence intervals around the point estimates for the treatment benefit likely overlap for eyes with and without RAP. As both the direction and the magnitude of the treatment effect cannot be distinguished between the subgroup of eyes with RAP vs those without, we conclude that the presence of RAP does not modify the favourable treatment outcomes achieved with PDT. The only analysis that suggests that RAP eyes have a less favourable response to PDT than eyes without RAP is the proportion of treated eyes that have a visual acuity of 20/200 or worse. However, RAP eyes started with lower levels of visual acuity, making it more likely that a greater proportion of eyes in this relatively limited group of 17 eyes would fall to this threshold compared with the group of 149 eyes without RAP.

\section{Summary of lessons learned from VIP trial with respect to RAP lesions}

The $10 \%$ prevalence of RAP among VIP trial participants was consistent with the reported estimates of the prevalence of this AMD variant. Prospective data on the natural history of these lesions did not identify any dramatic difference in outcomes for these eyes compared with similar eyes without RAP; although we cannot completely rule out a small interaction effect. A similar proportion of eyes with RAP, as compared with eyes without RAP, were assigned to each of the treatment groups such that comparisons of PDT treatment efficacy could be evaluated with reference to the presence of RAP. Vision outcomes with PDT did not seem to be influenced by the presence of RAP, and eyes with RAP treated with PDT did better than eyes allocated to the sham group. Thus, review of the VIP trial data dispels several myths about eyes with RAP. ${ }^{15}$

\section{Do other data corroborate the VIP experience?}

There are very few reports on the natural history of RAP lesions. A recent study characterized 16 eyes with RAP lesions that were observed for a variable length of time from 6 to 44 months. ${ }^{16}$ Mean visual acuity at presentation was 20/50 in this cohort and which then fell to 20/80 at the month 6 exam. Mean visual acuity at the last followup visit measured 20/100. Although there is no comparison group and the final visit covered a broad range for the duration of follow-up, these observations are not strikingly dissimilar from a typical neovascular AMD cohort. We know from earlier studies that lesion composition of AMD-associated CNV lesions affects the natural course. Generally speaking, eyes with occult lesions without a classic component (which reflects the majority of lesions that have RAP) can have quite a variable visual prognosis, and from this description, $\mathrm{CNV}$ with a retinal anastomosis to the CNV lesion appears to be no exception.

\section{Is Anti-VEGF therapy recommended for RAP lesions?}

Several recent reports suggest that anti-VEGF therapy is a promising treatment modality for eyes with RAP lesions. Given that anti-VEGF therapy has become the standard of care for the majority of neovascular AMD lesions, it comes as no surprise that various groups would evaluate their experience with either ranibizumab or bevacizumab for eyes with RAP lesions. In addition, there is a plausible biological rationale to pursue antiVEGF treatments for these lesions. Case-control studies of eyes with RAP lesions showed both early and late reductions in choroidal filling using ICG angiography in the eyes with RAP. This choroidal circulatory disturbance may in turn lead to hypoxia in the outer retina that may subsequently upregulate angiogenic growth factors such as VEGF. ${ }^{17}$ In addition, a recent immunohistochemical study identified VEGF in a RAP lesion from a cadaver eye. $^{18}$

Several small retrospective studies have described short-term results managing RAP lesions with bevacizumab. ${ }^{10,19,20}$ Each of these groups report stabilization or improvement of visual acuity in eyes with RAP lesions for a period of up to 3 months after initiation of intravitreal bevacizumab. Recently, Gharbiya et al..$^{21}$ reported 12-month outcomes in a previously treatment-naive cohort of 17 eyes followed prospectively while being managed with bevacizumab as a monotherapy. Each study eye received a monthly treatment of three doses followed by a ' $p r n$ ' or as needed, regimen of re-treatment at each of the monthly visits through to month 12 . Vision results were positive, with mean baseline ETDRS letter score rising from 40 letters (Snellen equivalent $\sim 20 / 42$ ) to 48 letters (Snellen equivalent $\sim 20 / 28$ ) at 12 months. In fact, three eyes, or $18 \%$, improved at least by 15 letters. Substantial reductions in optical coherence tomography (OCT) 
central thickness and cessation of fluorescein leakage corroborated the vision outcomes. ${ }^{21}$ These results are consistent with expectations, given that RAP is a variant of neovascular AMD, and it is very likely that RAP lesions were included in all the phase III studies evaluating ranibizumab for neovascular AMD. Once again, these data also refute the notion that RAP lesions may respond less well to the treatment when compared with other neovascular lesions.

Other groups of investigators have shared their positive experience using ranibizumab monotherapy for neovascular AMD lesions with RAP. ${ }^{22,23}$ A retrospective study describing 31 RAP lesions (some of which had received earlier PDT), managed with ranibizumab following a strictly prn regimen, reported outcomes 10-22 months after treatment initiation. During this variable length of follow-up, the average number of ranibizumab treatments was 5 (range 3-12). Comparison of the mean visual acuity at baseline and the mean visual acuity at the final visit for each patient suggested vision improvement (20/100 vs 20/63). Concomitant reductions in OCT-measured retinal thickness and cessation in fluorescein angiographic leakage were also apparent. ${ }^{23}$

As clinicians have been exploring combination therapies to address all forms of neovascular AMD, the same type of experimentation has been occurring in eyes with RAP lesions. The driving rationale to investigate combination therapy is to combine different mechanisms of action to stop the angiogenic sequence, and by doing so, to increase the efficiency of the treatment, as manifested by reduced treatment burden and maintenance of superior vision outcomes. A recent 9-month pilot study of eight treatment-naive RAP lesions, managed with a single application of ICG-guided PDT combined with monthly intravitreal bevacizumab for 3 months followed by a PRONTO style prn regimen ${ }^{24}$ with bevacizumab only thereafter, found favourable vision outcomes. ${ }^{25}$ Most of these eyes received the required three intravitreal injections and then required no further bevacizumab. Mean visual acuity improved from 20/100 to about 20/50, as 5 of 8 eyes gained at least 3 lines of acuity. Retinal thickness, as indicated on OCT, decreased from $372 \mu \mathrm{m}$ to $162 \mu \mathrm{m}$ during follow-up. Whether the benefit seen here is solely due to the bevacizumab or is attributed to the combination effects of bevacizumab plus PDT remains unknown. Thus far, limited information exists to compare combination treatment regimens with monotherapy regimens using anti-VEGF agents alone. In a limited prospective study of 37 eyes randomly assigned to ranibizumab monotherapy, PDT plus ranibizumab, or PDT plus intravitreal injection of $4 \mathrm{mg}$ triamcinolone, no difference in visual outcomes was noted at month 6, with most study eyes avoiding vision loss. However, this study had several limitations, including small numbers of participants within each treatment group, nonstandard Snellen visual acuities, and lack of masking of treatment assignment. ${ }^{26}$

\section{Summary}

'Retinal angiomatous proliferation' is a variant of neovascular AMD with a distinct clinical appearance. Multiple names have been used to describe this entity. In the absence of sequential histopathological specimens showing the evolution of RAP lesions, the debate will continue as to the origin of these lesions. Information from clinical trials suggests that the natural history of these lesions and their response to various $\mathrm{CNV}$ treatments may be similar to typical neovascular AMD lesions without a RAP component. ${ }^{15}$

\section{Conflict of interest}

The authors declare no conflict of interest.

\section{References}

1 Kuhn D, Meunier I, Soubrane G, Coscas G. Imaging of chorioretinal anastamoses in vascularized retinal pigment epithelial detachments. Arch Ophthalmol 1995; 113: 1392-1398.

2 Freund KB, Klais CM, Eandi CM, Ober MD, Goldberg DE, Sorenson JA et al. Sequenced combined intravitreal triamcinolone and indocyanine green angiography-guided photodynamic therapy for retinal angiomatous proliferation. Arch Ophthalmol 2006; 124: 487-492.

3 Hartnett ME, Weiter JJ, Gardts A, Jalkh AE. Classification of retinal pigment epithelial detachments associated with drusen. Graefes Arch Clin Exp Ophthalmol 1992; 230: 11-19.

4 Gass JD. Stereoscopic Atlas of Macular Diseases. 4th ed. CV Mosby: St Louis, 1997; 26-30.

5 Freund KB, Ho I, Barbazetto IA, Koizumi H, Laud K, Ferrara D et al. Type 3 neovascularization: the expanded spectrum of retinal angiomatous proliferation. Retina 2008; 28: 208-211.

6 Yannuzzi LA, Freund KB, Takahashi BS. Editorial: review of retinal angiomatous proliferation of type 3 neovascularization. Retina 2008; 28: 375-384.

7 Yannuzzi LA, Negrao S, Iida T, Carvalho C, RodriguezColeman H, Slakter $\mathrm{J}$ et al. Retinal angiomatous proliferation in age-related macular degeneration. Retina 2001; 21: 416-434.

8 Gass JD, Agarwal A, Lavina AM, Tawansy KA. Focal inner retinal hemorrhages in patients with drusen: an early sign of occult choroidal neovascularization and chrorioretinal anastomosis. Retina 2003; 23: 741-751.

9 Borrillo JL, Sivalingam A, Martidis A, Federman JL. Surgical ablation of retinal angiomatous proliferation. Arch Ophthalmol 2003; 121: 558-561.

10 Ghazi NG, Knape RM, Kirk TQ, Tiedeman JS, Conway BP. Intravitreal bevacizumab (Avastin) treatment of retinal angiomatous proliferation. Retina 2008; 28: 689-695. 
11 Verteporfin in Photodynamic Therapy Study Group. Verteporfin therapy of subfoveal choroidal neovascularization in age-related macular degeneration: two-year results of a randomized clinical trial including lesions with occult with no classic choroidal neovascularization- Verteporfin in Photodynamic Therapy report 2. Am J Ophthalmol 2001; 131: 541-560.

12 Hartnett ME, Weiter JJ, Staurenghi G, Elsner AE. Deep retinal vascular anomalous complexes in advanced age-related macular degeneration. Ophthalmology 1996; 103: 2042-2053.

13 Bottoni F, Massacesi A, Cigada M, Viola F, Muicco I, Staurenghi G. Treatment of retinal angiomatous proliferation in age-related macular degeneration. Arch Ophthalmol 2005; 123: 1644-1650.

14 Kusserow C, Michels S, Kollner T, Schmidt-Erfurth U. Impact of chorioretinal anastomosis on prognosis in photodynamic therapy [ARVO abstract]. Invest Ophthalmol Vis Sci 2001; 42: 512. Abstract 2761.

15 Bressler NM. Retinal anastomosis to choroidal neovascularization: a bum rap for a difficult disease. Arch Ophthalmol 2005; 123: 1741-1743.

16 Viola F, Massacesi A, Orzalesi N, Ratiglia R, Staurenghi G. Retinal angiomatous proliferation: natural history and progression of vision loss. Retina 2009; 29: 732-739.

17 Koizumi H, Iida T, Saito M, Nagayama D, Maruko I. Choroidal circulatory disturbances associated with retinal angiomatous proliferation on indocyanine green angiography. Graefes Arch Clin Exp Ophthalmol 2008; 2246: 515-520.

18 Monson DM, Smith JR, Klein ML, Wilson DJ. Clinicopathologic correlation of retinal angiomatous proliferation. Arch Ophthalmol 2008; 126: 1664-1668.

19 Meyerle CB, Freund B, Iturralde D, Spaide RF, Sorenson JA, Slakter JS et al. Intravitreal bevacizumab
(Avastin) for retinal angiomatous proliferation. Retina 2007; 27: 451-457.

20 Joeres S, Heussen FM, Treziak T, Bopp S, Joussen AM. Bevacizumab (Avastin) treatment in patients with retinal angiomatous proliferation. Graefes Arch Clin Exp Ophthalmol 2007; 245: 1597-1602.

21 Gharbiya M, Allievi F, Recupero V, Martini D, Mazzeo L, Gabrieli CB. Intravitreal bevacizumab as primary treatment for retinal angiomatous proliferation: twelve-month results. Retina 2009; 29: 740-749.

22 Lai TY, Chan WM, Liu DT, Lam DS. Ranibizumab for retinal angiomatous proliferation in neovascular age-related macular degeneration. Graefes Arch Clin Exp Ophthalmol 2007; 245: 1877-1880.

23 Konstantinidis L, Mameletzi E, Mantel I, Pournaras J-A, Zografos L, Ambresin A. Intravitreal ranibizumab (Lucentis) in the treatment of retinal angiomatous proliferation (RAP). Graefes Arch Clin Exp Ophthalmol 2009; 247: 1165-1171.

24 Lalwani GA, Rosenfeld PJ, Fung AE, Dubovy SR, Michels S, Feuer $\mathrm{W}$ et al. A variable-dosing regimen with intravitreal ranibizumab for neovascular age-related macular degeneration: year 2 of the PrONTO Study. Am J Ophthalmol 2009; 148: 1-3.

25 Lo-Giudice G, Gismondi M, De Belvis V, Clan R, Tavolato M, Galan A. Single-session photodynamic therapy combined with intravitreal bevacizumab for retinal angiomatous proliferation. Retina 2009; 29: 949-955.

26 Rouvas AA, Papakostas TD, Vavvas D, Vergados I, Moschos MM, Kotsolis A et al. Intravitreal ranibizumab, intravitreal ranibizumab with PDT, and intravitreal triamcinolone with PDT for the treatment of retinal angiomatous proliferation: a prospective study. Retina 2009; 29: 536-544. 